\title{
Common Barriers, Attitudes and Practices of Veterinary Practitioners Regarding Antimicrobial Resistance and Stewardship in Bangladesh
}

Md. Sahidur Rahman ${ }^{1,2 *}$, Nazifa Rafa ${ }^{1,3}$

1. One Health Center for Research and Action. Akbarshah, Chattogram-4207, Bangladesh

2. Livestock and dairy development project, Department of livestock services, Dhaka, Bangladesh

3. Environmental Sciences Program Asian University for Women. 20/A, MM Ali Road, Chattogram 4000, Bangladesh.

\section{Corresponding:}

sahid.dvm@gmail.com

+8801705727081

ORCID: https://orcid.org/0000-0002-2285-992X

\begin{abstract}
Usage of antimicrobials in veterinary practices has always been under scrutiny due to the perceived risk of resulting in antimicrobial-resistant pathogens. This creates the necessity for understanding the role of the prescriber group. Hence, we conducted a cross-sectional survey among veterinary practitioners from August to November 2019 in the Chattogram district of Bangladesh, aiming to assess the practitioner's perceptions regarding antimicrobial prescribing and antimicrobial resistance (AMR) issue. We collected responses from 100 veterinarians engaged in the treatment of the large animal, poultry, and pet animal through a self-administrated questionnaire. Proportions were calculated for categorical variables and the results are presented using visual aids. Our study revealed two key barriers - scarcity of enough information on antimicrobial used, and the lack of training in the proper prescription of antimicrobials. Participants recognized that prescribing too many varieties of antimicrobials and the use of an incomplete course of drugs as two very important causes for the development of antimicrobial resistance. In addition, prescription of inappropriate doses and incentives from pharmaceutical
\end{abstract}


companies were dubbed as important causes. We also found that along with clinical features and types of organisms, the availability of drugs in the local market and the economic conditions of farmers have potential impacts on the antimicrobials prescribing decision of the veterinarians. However, all participants recognized the emerging threats of AMR. Results suggested that capacity building of veterinarians and the maintenance of strong coordination are crucial in ensuring the proper engagement of veterinarians as the front-line fighters for tackling the AMR issue.

Keywords: Antimicrobial resistance, antimicrobial stewardship, barriers, perception, survey, veterinary practitioners

\section{Introduction}

Drugs that kill or suppress different types of disease-causing pathogens are called antimicrobials, of which antibiotics are an important group that acts against bacteria. When antimicrobials are introduced, they create a hostile environment for susceptible pathogen populations which facilitate selective survival and subsequently, the development of antimicrobial-resistant microorganisms known as superbug. Antimicrobial resistance (AMR) is an alarming global health crisis. Currently, Bangladesh is located in a high-risk zone assessed by the World Health Organization $(\mathrm{WHO})^{1}$. Infections caused by resistant microbes might be associated with increased treatment costs and unexpected morbidity and mortality. Considering the high impact of AMR, by 2050, livestock production is expected to fall by $11 \%$ in low-income countries $^{2}$. Indiscriminate use of too many antibiotics in livestock production as a growth promoter along with the prophylaxis and treatment of diseases has exacerbated the AMR problem. Statistics showed that more medically important antibiotics were used in the agriculture sector than in human medicine, and the rate was over 70 percent (by weight) in the USA ${ }^{3}$.

However, the implementation of policies can restrict this growing risk ${ }^{2}$. As a result, in May 2017, Bangladesh introduced its national action plan (BNAP) for antimicrobial resistance containment based on the global action plan endorsed by WHO. BNAP prioritizes the prudent use of antimicrobials by the establishment and implementation of standard treatment guidelines and antibiotic stewardship protocol in both the veterinary and the human health sectors ${ }^{4}$. Although the BNAP aligns well with the WHO global action plan (GAP), gaps in financing, 
antimicrobial stewardship, and operational and monitoring and evaluation frameworks still exist $^{5}$.

Despite the efforts to promote the culture of prudent use of antimicrobials, antimicrobialresistant microbes (superbugs) continue to emerge. The veterinary sector is one of the major sector of concern that can lead to constrained the proliferation of antimicrobial-resistant microbes and play a key role in safeguarding both human and animal health. As a result, the fourth objective of BNAP focuses on the optimized use of antimicrobials, throughout the veterinary sector, which is critical in curbing the AMR issue ${ }^{5}$. Thus, the success of the existing and future interventions requires the active participation of veterinarians as a key player for administering antimicrobials in animals.

Even though researchers have investigated different antimicrobial resistance patterns of microorganisms and resistant genes, there has been either no or very few studies focusing on the role of veterinarians regarding AMR in Bangladesh. Therefore, this study attempted to understand the common barriers, practices, and attitudes of field veterinarians from different disciplines towards antimicrobial uses and antimicrobial resistance. Results could be used as baseline evidence to advocate the efficient interventions and action plans to tackle the AMR issue through improving the antimicrobial stewardship practices in Bangladesh.

\section{Methodology}

\section{Study Design}

We carried out a cross-sectional survey among veterinarians who suggest antimicrobials in their everyday practices. Our target participants were all registered veterinarians involved in the treatment of large animals, poultry, and pet animals in the Chattogram district of Bangladesh, as well as interns and graduate students working in the teaching veterinary hospital under Chattogram Veterinary and Animal Sciences University (CVASU). Wildlife veterinarians were excluded from our study as they were very small in number and were less likely to prescribe antimicrobials in the study area. There were almost 150 veterinary doctors providing veterinary services in Chattogram, associated with government veterinary hospitals, private clinics, different private companies, or served as freelance-practitioners. Target students were almost 50 at the time of the survey. We employed purposive sampling to enroll participants from the target groups based on their willingness to participate. We drafted a semi-structured questionnaire that 
inquired into the barriers, attitudes, and practices of veterinarians in the English language and provided to each practitioner through direct meetings between the period of August to October in 2019. Before the interview, the purpose of the study was informed to all participants and oral consent was obtained from each of them. No incentives were given for participating in the survey.

\section{Data Collection Tool}

The survey questionnaire was designed in collaboration with experienced veterinarians, relevant researchers, and epidemiologists. The questionnaire was intended to extract information on demographics, barriers and factors influencing antimicrobial prescription, perception of threat, causes of AMR, commonly used antimicrobials beliefs and attitudes towards antimicrobial resistance and stewardship. Data collection was performed with the assistance of a group of trained veterinary students working as research intern under "One Health Center for Research and Action”. Most of the questions contained Likert-type responses measured on a 5point scale from very important to least important but the questions regarding the perception of threat included strongly agree to strongly disagree. 'Unsure', 'neutral' and 'other' options were also provided in different close-ended questions.

\section{Ethical Consideration}

This study was carried out in accordance with the recommendations of the Chattogram Veterinary and Animal Sciences University's ethics committee

\section{Data Analyses}

The responses were entered in Microsoft Excel 2010 (Microsoft Corporation, Redmond, Washington, USA) then coded and analyzed using Statistical Package for Social Science (SPSS) software version 25. We performed descriptive statistics for determining the frequency distributions of items within each question and visualize the results.

\section{Results}

Among total veterinary participants, $32 \%$ were engaged in the private sector, $23 \%$ in the government livestock department, $18 \%$ declared themselves as freelance-practitioners, $14 \%$ were interning doctors, and rest $13 \%$ were duty doctors in different institutional or private vet clinics. The majority of participants (89\%) were male and $11 \%$ were female. $61 \%$ of respondents acknowledged they have faced difficulties in selecting the correct antimicrobials during 
prescription. $63 \%$ of them also pointed out the scarcity of adequate sources of information on the use of antimicrobials and $67 \%$ claimed that they did not receive any formal training on antimicrobial prescribing within the last one year.

The perceptions of the participants regarding the contribution of different causes for the development of AMR have been presented in Figure 1. Prescription of wide varieties of antimicrobials and the ignorance of farmers to complete the full course of the drugs were two very important factors, selected by the respondents of the study, which leads to the rise of AMR.

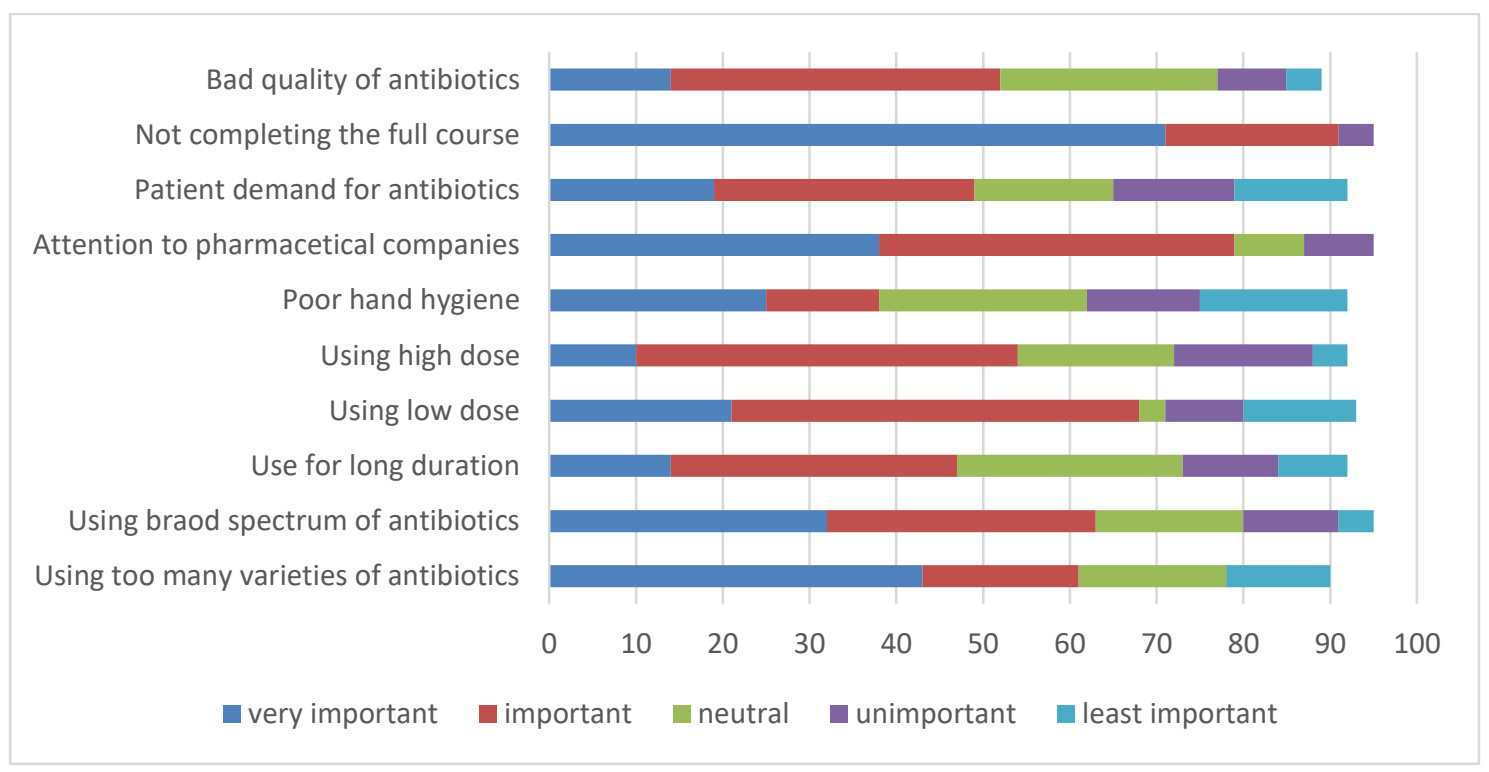

Figure 1: Perception of veterinarians to the most and least important causes of antimicrobial resistance

Figure 2 represented that amoxicillin, oxytetracycline, ciprofloxacin, and gentamicin were extensively prescribed by all groups of veterinarians. Ceftriaxone, penicillin, and streptomycin were mainly prescribed for large and small animals. Whereas, Colistin, tylosin, and levofloxacin were extensively used Poultry practices. 


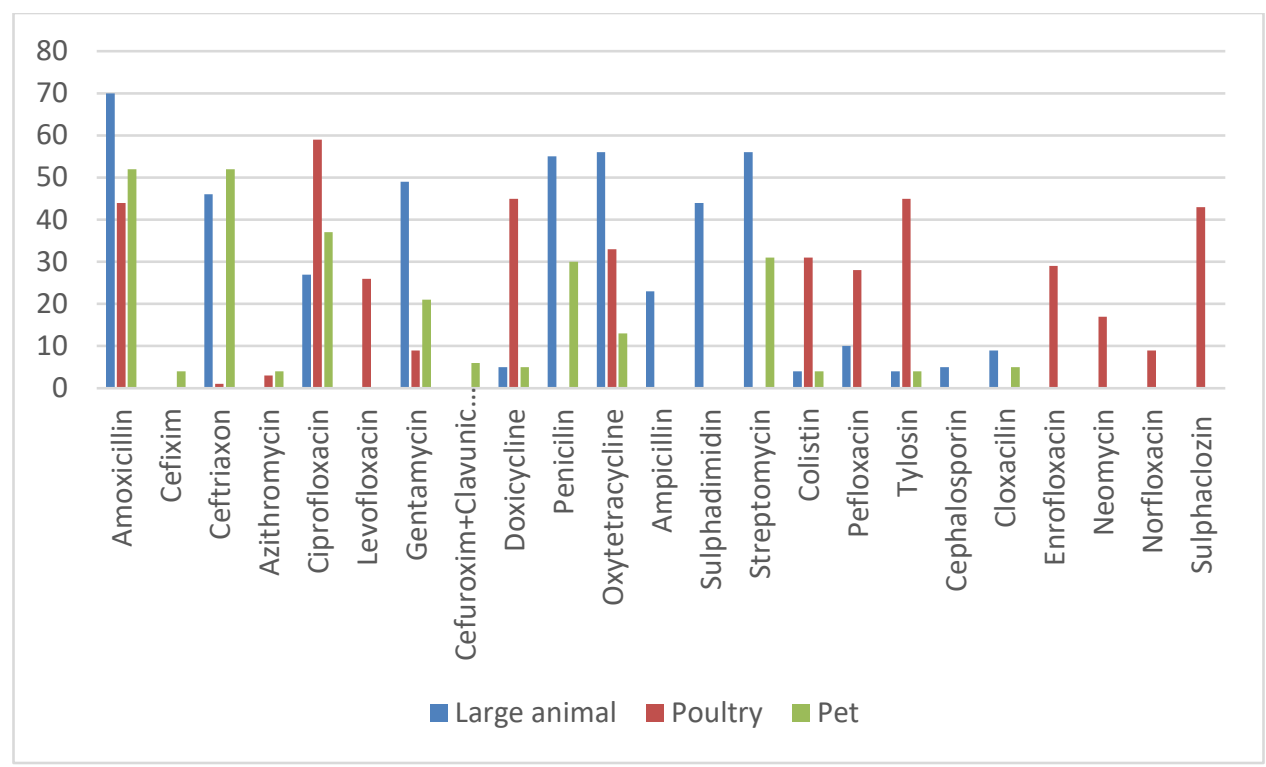

Figure 2: List of commonly prescribed antimicrobials in large animals, poultry, and pet animals

We also elucidated different factors influencing the selection of antimicrobials by the participants. Different clinical and non-clinical factors recognized by the participants to be paramount in their prescription decisions are shown in Figure 3.

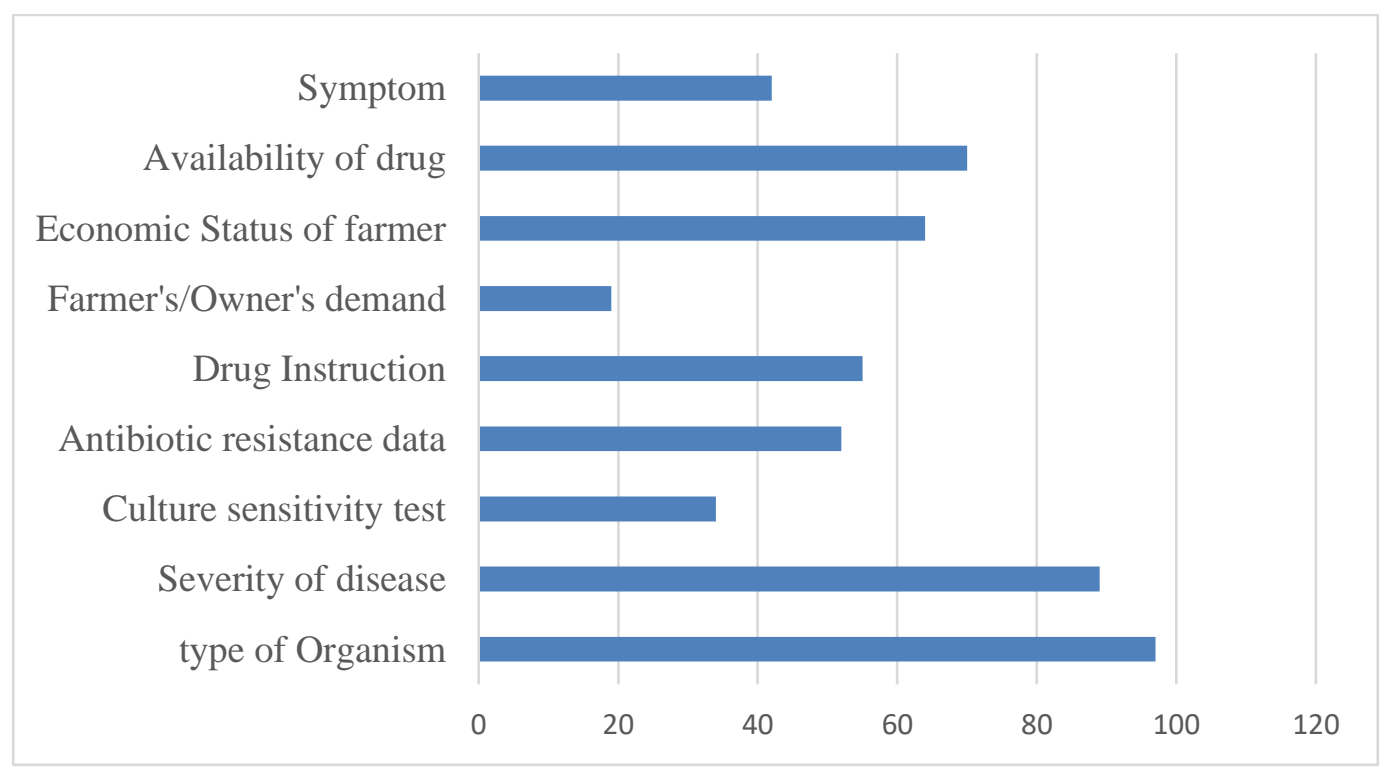

Figure 3: Factors considered by veterinarians during the prescription of antimicrobials

All respondents of this study either strongly agreed or agreed with the statement that AMR is a global problem, a national problem as well as problematic for their own daily practices. $33 \%$ of 
the respondents have regularly suggested the farmers for keeping records of drugs they used and only $10 \%$ advised to maintain the withdrawal period of the antibiotics after administered.

\section{Discussion}

Results disclosed common barriers that impact the prescribing decision of a veterinarian. Respondents of this survey stated that that they struggled with the selection of the right antibiotics in their daily practices and claimed that there was a scarcity of adequate information sources to guide their antimicrobials prescribing. This finding highlighted the professional communication gaps that lead to inappropriate use of antimicrobials, especially by new practitioners. The findings of Hardefeldt et al. (2018) ${ }^{6}$ echoed similar results as it mentioned the lack of antimicrobial prescribing policies and found only $28 \%$ of respondents followed available guidelines. The European Platform for Responsible Use of Medicines in Animals (EPRUMA), Australasian Infectious Disease Advisory Panel guidelines and the British Small Animal Veterinary Association guidelines are serving as the source of information for supporting the prescribers in Europe and Australia. It posed as a challenge for local regulatory bodies to provide uniform guidelines, training and other means of information sources. Previous studies conducted in other countries also expressed the necessity of guidelines for responsible antimicrobial prescription $^{7,8}$. Training modules should be field-oriented and interactive as traditional teaching methods and study materials often fail to create long-lasting impacts ${ }^{9}$. However regular dissemination of updated data and monitoring field practice is also highly recommended to take control over the prescribing approach.

The prescribers recognized the possible causes of AMR and ranked them based on the role of each cause. They mostly represented the causes related to their own practices in "very important" and "important" categories as well as the farmers' role by not completing the full course of antimicrobials as a very important factor. In addition, they also claimed the role of pharmaceutical companies for encouraging the use of more drugs as an important cause of AMR development. Competitive marketing policies create pressure on the prescribers' decisions for using antimicrobials at the cost of patients, stated by a similar study ${ }^{10}$. Literature also determined that pharmaceutical companies could regulate the antimicrobial prescribing decisions over half of the farm animal practitioners ${ }^{11}$. The low quality of the drugs available in the market was also 
indicated as an important factor by some of the participants. Results revealed the diversity of the problem and the contribution of different stakeholders from production to the application of antimicrobials. Simultaneously, it creates a demand for multisectoral engagement and multidimensional strategies to challenge the threat.

Participants were found to be using different critically important antimicrobials (CIA) of human health in veterinary practice, especially gentamicin, streptomycin, neomycin, cefixime, ceftriaxone, azithromycin, amoxicillin, and ampicillin. Moreover, the highest priority critically important antimicrobials (HP-CIAs), namely quinolones, macrolides, and $3^{\text {rd }}$ and $4^{\text {th }}$ generation cephalosporins, were also frequently used in poultry and large animals. WHO does not recommend the use of HP-CIAs as the first choice of antimicrobials, and suggests their uses be reserved for conditions where other classes of antimicrobials are ineffective or response is poor $^{12}$. Moreover, the American Veterinary Medical Association called for abstainment from the use of aminoglycosides, including gentamicin, in food-producing animals because the FDA has not been able to establish a safe withdrawal time for cattle. In addition, the extra-label use of gentamicin is considered as illegal in cattle ${ }^{13}$

We explored a set of different factors that influenced a veterinarian's daily practices of antimicrobial prescribing has been explored by this study. Other than the types of organisms and clinical signs, a number of non-clinical factors also contribute to motivating a veterinarian's drug choice during treatment- primarily the availability of drugs in the local market and the economic statuses of the farmers. In fact, a previous study had found the considerable effects of psychological and contextual factors over the practices of antimicrobial stewardship by veterinarians ${ }^{14}$. The findings of this study are also concordant with the existing literature which stated the decisions of a veterinarian are influenced by the expectations of farmers and farm infrastructure 7,15 . Similarly, our participants also reported the owner's demands as impacting their decisions during prescribing antimicrobials. The situation is also quite prevalent in human medicine practices ${ }^{16,17}$. These factors stand as some of the barriers to the application of antimicrobial stewardship and could potentially divert the prescriber's behavior away from the standard approach.

The respondents were also concerned about the extent of negative impacts and risks created by AMR globally, nationally, as well as in their own practices, a concern which was also 
found to be common in another study conducted on doctors, veterinarians, and dentists ${ }^{18}$. This awareness indicates the alertness and scope of veterinarians regarding active participation for a better solution to this crisis.

The survey was not based on a random selection of practitioners, which may have introduced bias. However, to the best of the knowledge of us this is the first survey among Bangladeshi veterinarians pertaining to AMR. As a result, there was a scarcity of enough local literature to compare the findings. Although we included only participants from the Chattogram district, the results could be used as the reflection of overall status of veterinarians of the country. Our findings might facilitate the regulatory bodies to optimize strategies focusing on the veterinarian's role and for proper implementation of the national action plan.

\section{Conclusion}

This study provides a systematic understanding of the perceptions of veterinarians regarding the overall aspects of AMR, as well as an understanding of their perceived roles as antimicrobial stewards. The findings might help to explore the challenges and opportunities in tackling the AMR issue to ensure the maximum contribution of veterinarians as frontline fighters towards the success of the national action plan.

\section{Funding}

This research did not receive any specific grant from funding agencies in the public, commercial, or not-for-profit sectors.

\section{Conflict of Interest Statement}

The authors declare that the research was conducted in the absence of any commercial or financial relationships that could arise as a potential conflict of interest.

\section{Acknowledgements}


We thank the research interns of One Health Center for Research and Action for their assistance in data collection, especially Dr. Muktadir Billah Reza, Dr. Zahan Ara and Md Mynul Islam. We also thank all the veterinarian practitioners who have given their time to participate in the survey.

\section{References}

1. Chereau F, Opatowski L, Tourdjman M, Vong S. Risk assessment for antibiotic resistance in South East Asia. BMJ. 2017 Sep 5;358:2-8.

2. World Bank. DRUG-RESISTANT INFECTIONS A Threat to Our Economic Future [Internet]. Washington, DC; 2017 [cited 2020 May 24]. Available from: www.worldbank.org

3. O’Neill J. TACKLING DRUG-RESISTANT INFECTIONS GLOBALLY: FINAL REPORT AND RECOMMENDATIONS THE REVIEW ON ANTIMICROBIAL RESISTANCE [Internet]. London, UK; 2016 [cited 2020 May 24]. Available from: https://amr-review.org/sites/default/files/160525_Final paper_with cover.pdf

4. DGHS/MoHFW. Ministry of Health \& Family Welfare National Action Plan Antimicrobial Resistance Containment in Bangladesh. 2017 [cited 2020 May 24]; Available from: https://www.flemingfund.org/wpcontent/uploads/d3379eafad36f597500cb07c21771ae3.pdf

5. Orubu ESF, Zaman MH, Rahman MT, Wirtz VJ. Veterinary antimicrobial resistance containment in Bangladesh: Evaluating the national action plan and scoping the evidence on implementation. J Glob Antimicrob Resist. 2020 Jun 1;21:105-15.

6. Hardefeldt LY, Gilkerson JR, Billman-Jacobe H, Stevenson MA, Thursky K, Bailey KE, et al. Barriers to and enablers of implementing antimicrobial stewardship programs in veterinary practices. J Vet Intern Med [Internet]. 2018 May 1 [cited 2020 May 27];32(3):1092-9. Available from: http://doi.wiley.com/10.1111/jvim.15083

7. De Briyne N, Atkinson J, Pokludová L, Borriello SP, Price S. Factors influencing antibiotic prescribing habits and use of sensitivity testing amongst veterinarians in Europe. 
Vet Rec. 2013 Nov 16;173(19):475.

8. Ungemach F, Müller-Bahrdt D, Of GA-IJ, 2006 U. Guidelines for prudent use of antimicrobials and their implications on antibiotic usage in veterinary medicine. Elsevier [Internet]. 2006 [cited 2020 May 26];33-8. Available from: https://www.sciencedirect.com/science/article/pii/S1438422106000634

9. Gray J. CHANGING PHYSICIAN PRESCRIBING BEHAVIOUR A CSCP LECTURE PRESENTED AT THE 2005 CANADIAN THERAPEUTICS CONGRESS [Internet]. Journal of population Therapeutics and Clinical Pharmacology. 2006 [cited 2020 May 27]. Available from: https://www.jptcp.com/index.php/jptcp/article/view/111

10. Coyne, L.A., Latham, S.M., Dawson, S., Donald, I.J., Pearson, R.B., Smith, R.F., Williams, N.J. and Pinchbeck GL. Antimicrobial use practices, attitudes and responsibilities in UK farm animal veterinary surgeons. Prev Vet Med [Internet]. 2018 [cited 2020 May 25];(161):115-26. Available from: https://www.sciencedirect.com/science/article/pii/S0167587718304999

11. Postma M, Speksnijder D, ... AJ-V, 2016 U. Opinions of veterinarians on antimicrobial use in farm animals in Flanders and the Netherlands. veterinaryrecord.bmj.com [Internet]. 2016 [cited 2020 May 26]; Available from: https://veterinaryrecord.bmj.com/content/vetrec/early/2016/06/16/vr.103618.full.pdf

12. WHO. Critically Important Antimicrobials for Human Medicine 5th Revision 2016 Ranking of medically important antimicrobials for risk management of antimicrobial resistance due to non-human use [Internet]. [cited 2020 Sep 9]. Available from: https://apps.who.int/iris/bitstream/handle/10665/255027/9789241512220eng.pdf;jsessionid=71FBC65E033489328B7176A5CE55BDC0? sequence=1

13. AVMA. Gentamicin sulfate, sulfamethoxazole used illegally | American Veterinary Medical Association [Internet]. [cited 2020 Sep 9]. Available from: https://www.avma.org/javma-news/2004-08-01/gentamicin-sulfate-sulfamethoxazoleused-illegally

14. Golding SE, Ogden J, Higgins HM. Shared goals, different barriers: A qualitative study of 
UK veterinarians' and farmers' beliefs about antimicrobial resistance and stewardship. Front Vet Sci. 2019;6(APR).

15. McDougall S, Compton CWR, Botha N. Factors influencing antimicrobial prescribing by veterinarians and usage by dairy farmers in New Zealand. N Z Vet J. 2017 Mar 4;65(2):84-92.

16. Tonkin-Crine S, Yardley L, Antimicrobial PL-J of, 2011 U. Antibiotic prescribing for acute respiratory tract infections in primary care: a systematic review and metaethnography. academic.oup.com [Internet]. 2011 [cited 2020 May 27];66(10):2215-23. Available from: https://academic.oup.com/jac/article-abstract/66/10/2215/730828

17. Hockenhull J, Turner AE, Reyher KK, Barrett DC, Jones L, Hinchliffe S, et al. Antimicrobial use in food-producing animals: A rapid evidence assessment of stakeholder practices and beliefs. Vet Rec. 2017;181(19):510.

18. Zhuo A, Labbate M, Norris J, Gilbert G, Open MW-B, 2018 U. Opportunities and challenges to improving antibiotic prescribing practices through a One Health approach: results of a comparative survey of doctors, dentists. bmjopen.bmj.com [Internet]. 2018 [cited 2020 May 25];8(3):e020439. Available from: https://bmjopen.bmj.com/content/8/3/e020439.abstract 